\title{
Plausibility of Image Reconstruction Using a Proposed Flexible and Portable CT Scanner
}

\author{
Jeff Orchard ${ }^{*}$, , Hwa Young Kim ${ }^{1}$ and John T.W. Yeow ${ }^{2}$ \\ ${ }^{I}$ David R. Cheriton School of Computer Science, ${ }^{2}$ Department of Systems Design Engineering, University of Waterloo, \\ Waterloo, ON N2L 3G1, Canada
}

\begin{abstract}
Abstarct: The very hot and power-hungry x-ray filaments in today's computed tomography (CT) scanners constrain their design to be big and stationary. What if we built a CT scanner that could be deployed at the scene of a car accident to acquire tomographic images before moving the victim? Recent developments in nanotechnology have shown that carbon nanotubes can produce x-rays at room temperature, and with relatively low power needs. We propose a design for a portable and flexible CT scanner made up of an addressable array of tiny x-ray emitters and detectors. In this paper, we outline a basic design, propose a strategy for reconstruction, and demonstrate the feasibility of reconstruction using experiments on a software simulation of the flexible scanner. These simulations show that reconstruction quality is stable over a wide range of scanner geometries, while progressively larger errors in the scanner geometry induce progressively larger errors. We also raise a number of issues that still need to be overcome to build such a scanner.
\end{abstract}

Keywords: Computed tomography, reconstruction, nanotechnology, PET.

\section{BACKGROUND}

Current clinical computed tomography (CT) scanners are rather large and therefore stationary. Their basic design includes a single $\mathrm{x}$-ray source that traverses a circular trajectory around the patient, and detectors on the opposite side of the patient sense the transmitted x-rays. The x-ray source requires a lot of power and is very hot, contributing to the reason why CT scanners are designed to be stationary.

Recently, carbon nanotubes (CNTs) have been used to generate $\mathrm{x}$-rays at room temperature, consuming far less power than conventional x-ray emitters [1]. Moreover, work is being done to integrate these tiny $\mathrm{x}$-ray devices into a flexible array such that each emitter can be individually pulsed [2]. While such an "x-ray blanket" could have many applications, we focus here on the concept of using the array, in conjunction with $\mathrm{x}$-ray detectors, as a flexible and portable CT scanner. In this paper, we outline a design concept for a flexible CT scanner, and use computer simulations to demonstrate the feasibility of using the data from the scanner to reconstruct tomographic images, despite the scanner's irregular geometry. In the paper's Conclusion section (section 5), we outline several hurdles that must be overcome in order to build a working prototype of the scanner.

Since their discovery in 1991 [3], carbon nanotubes have been vigorously investigated to understand their behaviour and devise new applications. The field emission property of CNTs is particularly interesting to the medical imaging community. In traditional $\mathrm{x}$-ray generation, a thermionic emission process is used, requiring a lot of energy and intense heat to release $\mathrm{x}$-rays. By contrast, the field emission process

*Address correspondence to this author at the David R. Cheriton School of Computer Science, University of Waterloo, Waterloo, Ontario N2L 3G1 Canada; Tel: (519) 888-4567, Ext. 35037; Fax: (519) 885-1208; E-mail: jeff.orchard@uwaterloo.ca releases electrons at room temperature in the presence of a strong electric field. Field emission from CNTs was first reported in 1995 [4-6], and since then has been developed for many applications, such as field emission displays, gas discharge tubes, nanolithography system, electron microscopes, lamps, and $\mathrm{x}$-ray tube sources [7, 8]. The exceptional field emission property of CNTs is due to their geometry, high thermal conductivity, and chemical stability. The current density $(J)$ obtained from field emission follows the Fowler-Nordheim equation [9],

$$
J=\frac{B E^{2}}{\Phi} \exp \left(-\frac{C \Phi^{3 / 2}}{E}\right)
$$

where $E$ is the electric field, $\Phi$ is the work function of the cathode material, and $B$ and $C$ are constants. The high aspect ratio of the CNTs serves to amplify the electric field. Even with a small work function of 4 to $5 \mathrm{eV}$, CNTs can be excellent field emitters [10].

The advent of CNT-based x-ray emitters has created the possibility of miniature $x$-ray devices. Experiments by Zhang et al., $[1,11]$ have produced $x$-ray images from a small bank of CNT emitters, each spaced just over a centimetre apart. Smaller x-ray generators could potentially be interleaved with detectors. In this architecture, an active subset of $\mathrm{x}$-ray emitters would produce multiple fan beams from different angles. Each x-ray emitter is digitally addressable and can be pulsed to mimic the mechanical rotation of a CT gantry system. Moreover, electronic switching enables multiplexing, allowing multiple $x$-ray images to be acquired simultaneously using the modulation/demodulation technique, as proposed by Zhang et al. [11].

Carbon nanotubes have recently been proposed for CT scanners. Quan et al., [12] proposed two designs for CT scanners based on pulsed CNT emitters. Their designs include square and hexagonal arrangements of flat-panel 
emitter and detector arrays positioned across from one another. The emitters are pulsed, one at a time, and the entire array of detectors is sampled for each emitter pulse. They used computer simulations to demonstrate the feasibility of reconstructing images using these geometries.

While these designs are an interesting departure from the standard clinical CT scanner, none are proposed as a flexible or portable device. Here, we focus on a design concept that is intended to be versatile, flexible and portable. We concede that a number of practical hurdles exist. Nonetheless, we put forth our proposal in the hopes that these issues are someday solved, or that the ideas presented here lead to a design that is more conducive to production.

\section{METHODS}

\subsection{Scanner Geometry}

The new CT scanner using CNTs has an array of emitters and detectors embedded into a flexible sheet, and is intended to be wrapped around an object to collect x-ray projections. Since the device is still under development, we report here on a project involving a software simulation of a prototype of the virtual CT scanner and use simulations to demonstrate the $\mathrm{CT}$ reconstruction algorithm.

The simulated CT scanner has a $360 \times 19$ rectangular grid of interleaved emitters and silicon photodiode detectors arranged in a checker-board pattern. Each detector and emitter is assumed to inhabit a square measuring $2.35 \mathrm{~mm}$ on a side. Thus, if the scanner sheet were bent into a cylinder, as shown in Fig. (1), the total circumference of the scanner would be $84.6 \mathrm{~cm}$ and its radius $13.5 \mathrm{~cm}$. Fig. (1) shows both the cylindrical and flat configurations of the flexible scanner. In the cylindrical configuration, the center of each emitter's cone beam passes through the scanner's $z$-axis. We will refer to this perfectly circular scanner configuration as the baseline. We then simulate deformation of the scanner using a displacement field defined by a $5 \times 4$ grid of control points located on the flexible scanner sheet, as described in section 2.3. By moving these control points, we simulate different scanner geometries in our experiments.

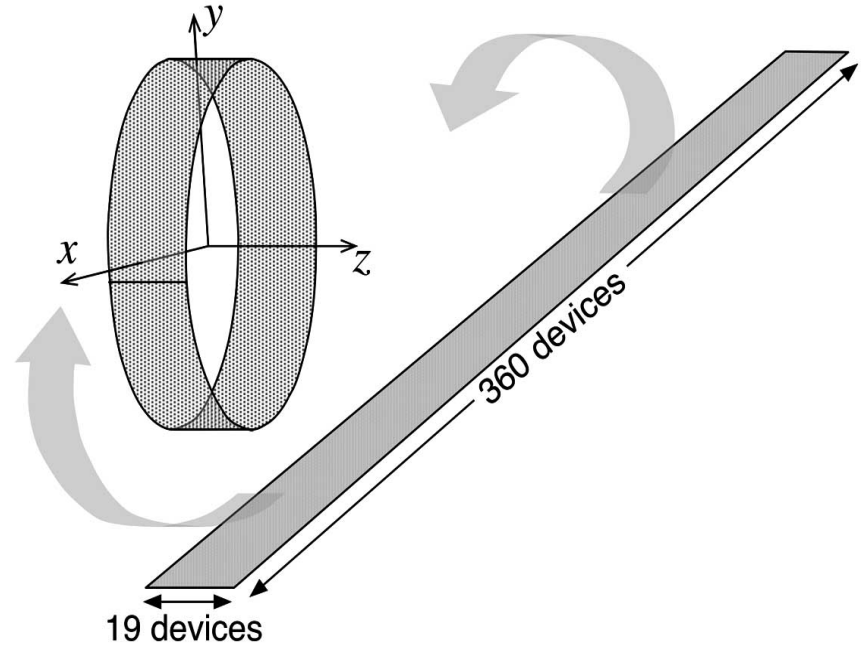

Fig. (1). Rectangular sheet containing a $360 \times 19$ grid of emitters and detectors, showing its cylindrical form and its flat form [13].

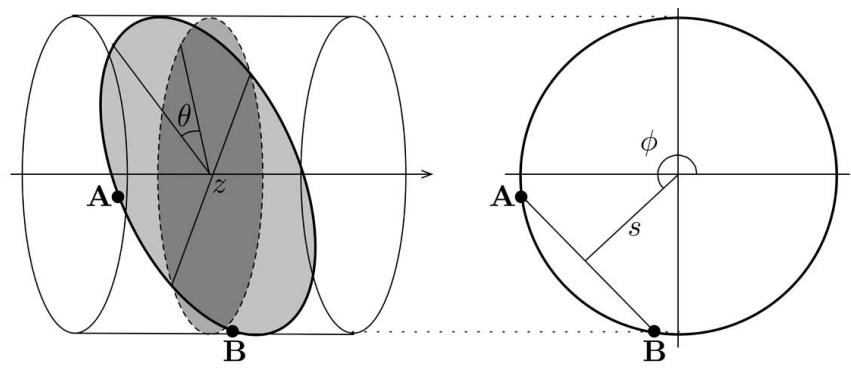

Fig. (2). Parameterization of the 4-D x-ray transform space, $(s, \phi, z, \delta)$, where $\delta=\tan \theta[13]$.

\subsection{Reconstruction}

Currently, most CT scanners use reconstruction methods that are specially designed to suit their fixed circular geometry. Moreover, scanner geometry is often designed to help the reconstruction process. In contrast, our portable and flexible scanner does not have a fixed circular geometry. Consequently, each scan might involve a different geometry. The flexible nature of the scanner makes the reconstruction more challenging.

We will assume that the 3-D position of each emitter and detector is known, since this information is required for the reconstruction (we return to this point in section 4). If we pulse one emitter at a time, then we know the ray path corresponding to each measurement of each detector. Hence, every ray sum can be put into the appropriate geometrical context and used in reconstruction.

A reconstruction method for a flexible 2-D (planar) scanner was presented in [14]. The collection of ray sums acquired by the virtual 2-D scanner did not fall onto a regular grid. Rather, the ray sums were used to populate the 2-D Radon transform (sinogram), and the collection of points was resampled onto a regular grid. The resulting gridded sinogram could then be fed into a standard 2-D reconstruction method such as filtered back-projection.

Our approach is similar for the 3-D case. Like in the 2-D case, these ray sums are used to populate the corresponding $\mathrm{x}$-ray transform space. However, unlike 2-D CT reconstruction, the x-ray transform for a 3-D scanner is 4-dimensional. Fig. (2) shows a common parameterization of the 4-D space, $(s, \phi, z \delta)[15]$, as well as a single ray that travels from $\mathbf{A}$ to $\mathbf{B}$ in a $3-\mathrm{D}$ cylindrical scanner. The logarithm of the intensity of the transmitted x-ray beam can be modeled by the Radon transform,

$$
p(s, \phi, z, \delta)=\int_{l_{A}}^{l_{B}} f(s \cos \phi-l \sin \phi, s \sin \phi+l \cos \phi, z+l \delta) \mathbf{d} l
$$

where $f$ is the 3-D intensity function being imaged, and $l_{A}$ and $l_{B}$ are the $l$-values corresponding to points $\mathbf{A}$ and $\mathbf{B}$ (where $l$ is in units of physical distance). In this 4-D x-ray transform space, $s$ and $\phi$ correspond to the polar coordinates of the ray when projected onto a transaxial plane, $z$ is the average of the $z$-values for $\mathbf{A}$ and $\mathbf{B}$, and $\delta$ represents the tangent of the angle between the transaxial plane and the plane containing points $\mathbf{A}, \mathbf{B}$ and $(0,0, z)$.

This projection data is used to reconstruct a CT volume by first resampling the irregular projections onto a regular grid in the 4-D x-ray transform space. The four parameters 
are discretized so that the $s$-axis has 360 samples, the $\phi$-axis has 180 samples, the $z$-axis has 19 samples, and the $\delta$-axis has 19 samples. As a result, this 4-D space contains over 23 million grid elements. In order to efficiently reconstruct the volume, we need to estimate the 4-D x-ray transform on this regular grid. However, the location of the ray sums depend on the scanner's geometry. Hence, we have to resample the ray sums onto our regular grid. We do this using Parzen windowing [16]. In short, each ray-sum sample is contained within a 4-D grid cell that is delineated by the 16 grid points in its corners. The sample is distributed among the 16 grid points, apportioned by proximity; a linear interpolation kernel is centred on the sample, and the kernel's value at each grid point dictates what fraction of the sample is contributed to that grid point. The sample density is similarly populated, so that -- by the end -- each grid point has a total intensity and a total weight. The $\mathrm{x}$-ray intensity value assigned to each grid point is a weighted average of all the ray sums that contributed to it, calculated by dividing the total intensity by the total weight.

To illustrate the method, Fig. (3) shows 16 adjacent grid points (one cell) in the 4-D x-ray transform space, depicted as two 3-D volumes at neighboring $\delta$-values. Suppose that a ray sum has intensity value $X$, and that its location in the $\mathrm{x}$ ray transform space is $(\mathrm{s}, \phi, \mathrm{z}, \delta)=(8.4,31.1,2.3,0.7)$. The intensity value $X$ is partitioned and distributed among the nearest 16 grid locations, each location receiving a proportion according to the value of the linear-interpolation kernel centred at $(8.4,31.1,2.3,0.7)$. For example, the fractional weight assigned to the grid location of $(9,32,3,1)$ is the product $(0.4)(0.1)(0.3)(0.7)$. Hence, the bin that accumulates $\mathrm{x}$-ray intensity at location $(9,32,3,1)$ is incremented by intensity $(0.4)(0.1)(0.3)(0.7) X$, and that grid location's total weight is incremented by $(0.4)(0.1)(0.3)(0.7)$. Once all the ray sums have been distributed among the x-ray transform bins, the accumulated intensity value at each bin is divided by the total weight accumulated by the bin. This yields the weighted average of the ray sums that contributed to each grid element, and a good estimate of the x-ray transform that would have been acquired by a parallel-beam scanner.
Once the data is on a regular grid, the FORE-J method [17] is used to reconstruct a 3-D volume from the 4-D x-ray transform. The FORE-J method was recently used for inverse-geometry CT reconstruction [18]. Since the 3-D object is represented by 4-D data, it contains redundant information. A full description of the FORE-J reconstruction method is beyond the scope of this paper, but is described in detail in section 4.1 of [17]. In brief, the FORE-J method is a Fourier rebinning method that uses the redundant data in oblique and transaxial sinograms to improve the signal-tonoise ratio (SNR) in the reconstructed images. One of the advantages of the FORE-J method is that it requires interpolation of the data along only one dimension. Moreover, the output of the method is a stack of independent 2-D Radon transforms that can be reconstructed one slice at a time. We reconstruct those slices using 2-D filtered back-projection.

\subsection{Simulations}

To demonstrate the feasibility of reconstructing meaningful images from this flexible scanner design, we created a virtual CT scanner using a software simulator. Thus, the experiments done in this paper are based on data generated from the simulated CT scanner.

The construction of our virtual scanner is as follows. As described earlier, our baseline configuration is a perfectly cylindrical tube composed of 19 rings that encircle the $z$ axis, each ring containing 360 devices (180 emitters and 180 detectors). We also assume that each $x$-ray emitter generates a cone beam with a total apex angle of $120^{\circ}$. In this configuration, the centre of the cone beams pass through the $z$-axis.

A $5 \times 4$ grid of control points is used to deform this circular scanner. To create a testing scenario, we generate a set of 60 random numbers and use them as the $x, y$ and $z$ displacements for the 20 control points. For each direction, a bivariate cubic spline is fit through the displacement values. For example, consider the $x$-coordinate displacement only. Each control point in the $5 \times 4$ grid is assigned an $x$ -

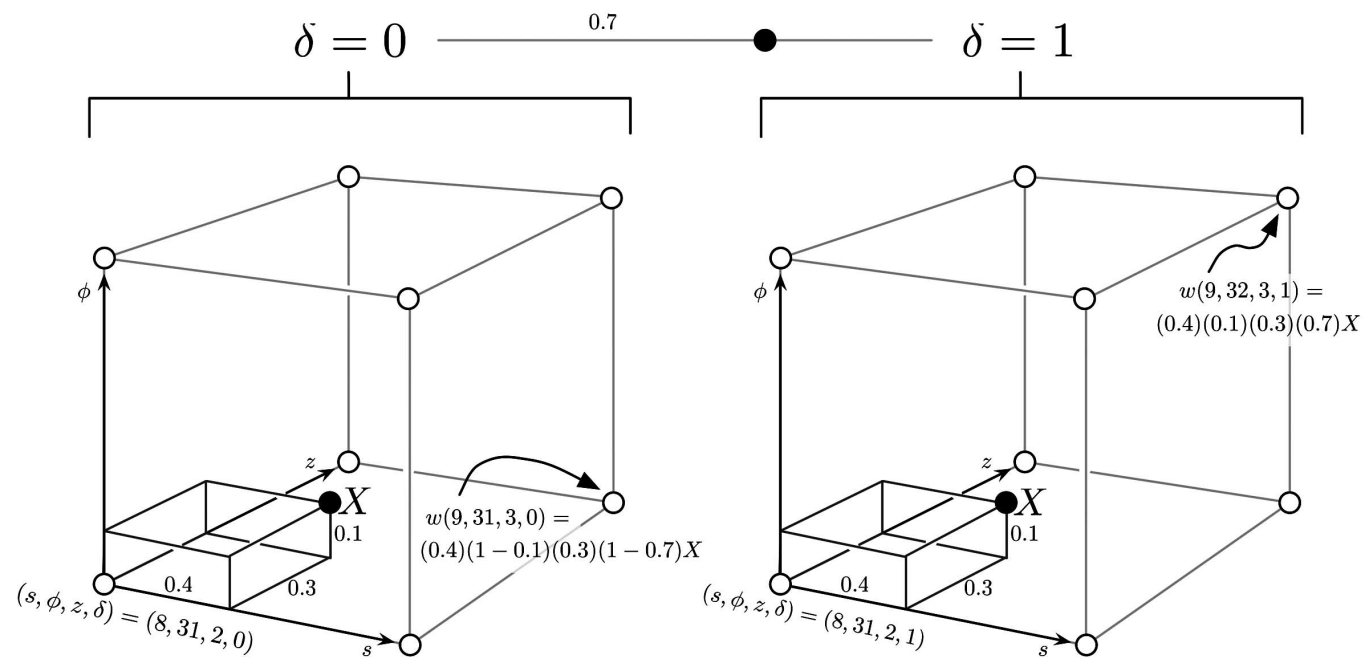

Fig. (3). Distribution of ray sums onto the 4-D x-ray transform grid. Each of the two cubes depicts 3 of the 4 dimensions, but at different $\delta$ values. The ray-sum value of $X$ is distributed among its 16 neighboring grid elements, apportioned according to proximity. In this example, the value at grid location $(s, \phi, z, \delta)=(9,32,3,1)$ is incremented by $(0.4)(0.1)(0.3)(0.7) X$, while the value at location $(s, \phi, z, \delta)=(9,31,3,0)$ is incremented by $(0.4)(1-0.1)(0.3)(1-0.7) X$. 
displacement value. A bivariate spline surface is fit to these displacement values; the surface dictates the $x$ displacement of every point on the scanner. The same process is done for the $y$ and $z$ components. Hence, the displacement of each emitter/detector is determined by these three splines. In this manner, we can generate different geometrical configurations of the CT scanner. Furthermore, the direction of each emitter's beam is determined by the normal to the spline surface. Hence, we assume we are given the position and orientation of each emitter/detector device.

Based on the positions of the emitters and detectors, we can determine which detectors are within the $\mathrm{x}$-ray cone for a given emitter.

In our experiments, we use two different test volumes, both $236 \times 330 \times 105$ voxels in dimension (approx. $1 \mathrm{~mm}$ isotropic voxels). One test volume is a CT volume of the neck from the Visible Female dataset (Visible Human Project, National Library of Medicine). The other test volume is a 3-D version of the Shepp-Logan phantom, created by Matthias Schabel. The virtual CT scanner is positioned around the anatomy and the $\mathrm{x}$-ray projections are computed by evaluating the line integral (2) along the straight lines between emitters and detectors. Ray sums are only computed for emitter/detector pairs where the detector rests within the emitter's x-ray cone beam. The ray sum is approximated by discretizing the line integral (2), sampling the imaging volume (using trilinear interpolation) every $0.3 \mathrm{~mm}$ along the ray path. We assume that our $\mathrm{x}$-rays are mono-energetic. Also, we assume that the angle of incidence does not affect the efficiency of a detector (this is a valid assumption if the geometry of the scanner is known, since the angle of incidence could then be computed and compensated for).

Once the projection data is resampled onto a regular grid, the last step is to apply the FORE-J method [17] to reconstruct the images. Fig. (4) shows the scanner in context with the Visible Female anatomy, as well as a reconstruction from the scanner after applying the FORE-J method. Note that since the scanner is circular in this case, resampling in the $z$ axis is not necessary to construct the sinograms. This ideal image is used as a gold standard for comparison in our experiments

In the next section, we outline three types of experiments designed to evaluate the robustness of the reconstructions generated from simulated data acquired from the flexible scanner. The first experiment tests the stability of our recons- tructions with respect to the degree of scanner deformation. The second test is designed to measure the effect of geometrical error -- where the geometrical information used during reconstruction is slightly different from the actual geometry that was used during data acquisition. The third type of experiment demonstrates the effect of missing data caused by either a reduced x-ray cone angle, or the scanner being too close to the patient.

To measure the effect of each factor, we compute the Normalized Mean Square Error (NMSE) between our reconstructed image and a gold standard image, within a region of interested (ROI, specified on the gold-standard image). The NMSE is given by

$$
E^{2}=\frac{\sum|g(x, y)-f(x, y)|^{2}}{\sum|f(x, y)|^{2}},
$$

where $g(x, y)$ is the reconstructed image, $f(x, y)$ is the gold standard image, and the summations are over all $(x, y)$ in the ROI. Before computing the NMSE, we perform a rigid-body least-squares registration between the images to remove any effect from misalignment.

\section{RESULTS}

\subsection{Irregular Geometry Test}

The first experiment tests how the reconstruction method behaves as the scanner geometry is irregularly deformed from its baseline circular configuration. The flexible CT scanner is intended to be bent around an object (body part) in an uncontrolled fashion. Naturally, we want the reconstructed images to be independent of the scanner geometry. Therefore, given the ideal reconstruction of the circular scanner as a gold standard (shown in Fig. (4d)), we aim to show that the degree of scanner deformation has little impact on image quality.

To simulate irregular CT geometries, we randomly generated a set of control point offsets; recall that we perturb our virtual scanner from its baseline shape by manipulating a $5 \times 4$ grid of control points. We then generate different scanner geometries by multiplying these offsets by a deformation factor, $d$. When $d$ is zero, our scanner is circular. As $d$ increases, the scanner is progressively deformed. Changing the deformation factor by 5 moves the devices by an average of $9.3 \mathrm{~mm}(\min .=0.97 \mathrm{~mm}, \max .=18.6 \mathrm{~mm})$. Fig. (5)

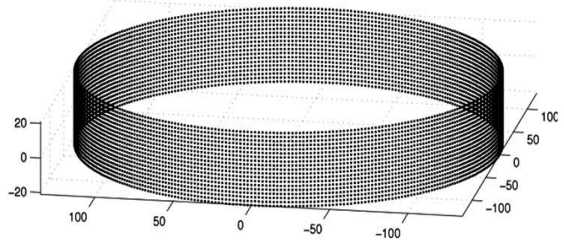

(a)

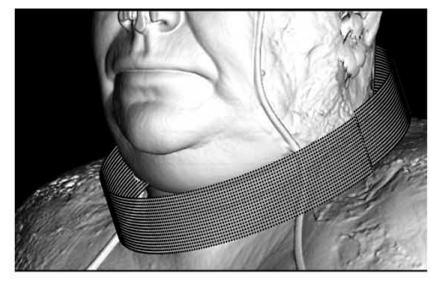

(b)

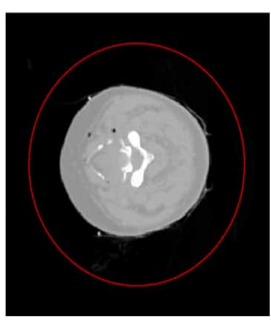

(c)

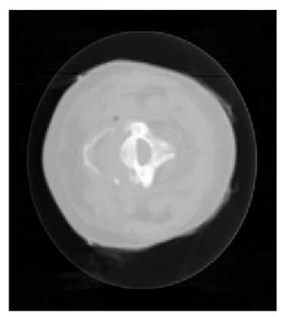

(d)

Fig. (4). Baseline scanner configuration. (a) shows the 3-D shape of the scanner, (b) shows the scanner around the neck (rendered using VolumeJ [19]), (c) shows a cross-section of the scanner in the context of the anatomy, and (d) is a reconstructed image from the baseline configuration. The graylevel maps for (c) and (d) were set so that the lowest intensity was mapped to black and the highest to white. We used the same convention for all the other $\mathrm{CT}$ images in this paper. 


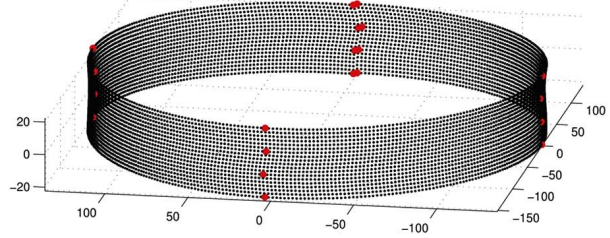

(a) $d=5$

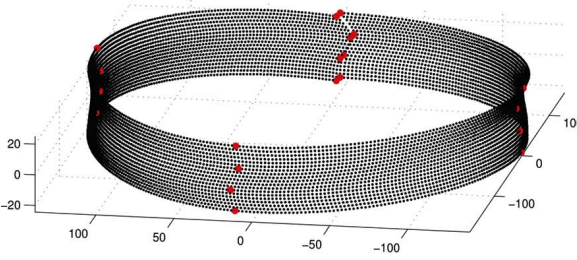

(c) $d=15$
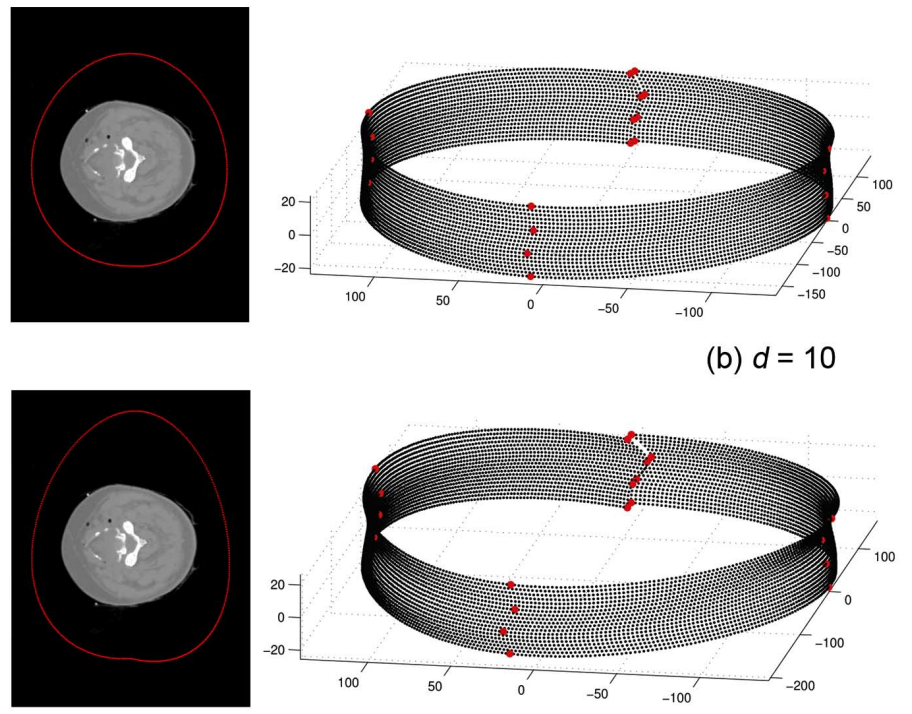

(b) $d=10$

(d) $d=20$
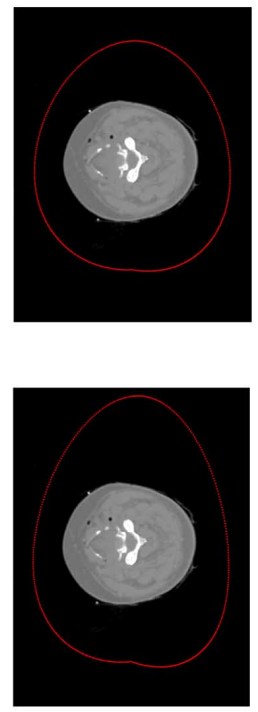

Fig. (5). Deformed CT scanner configurations depicted in 3-D and 2-D, using deformation factors $d=5,10,15$, and 20 .

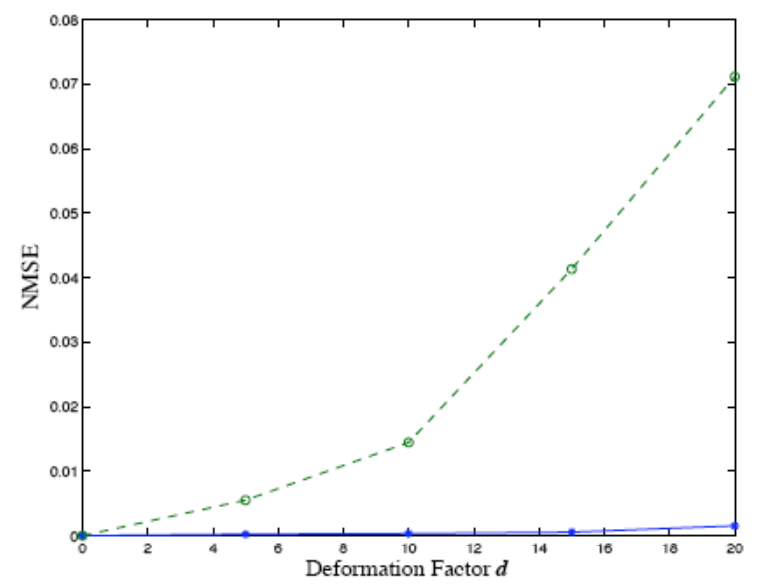

(a)

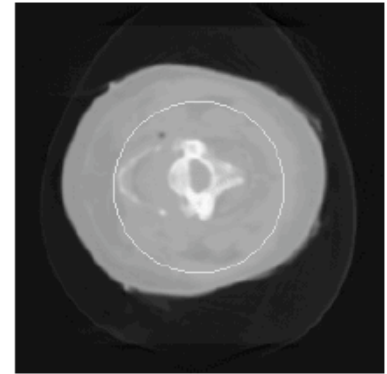

(b) $d=5$

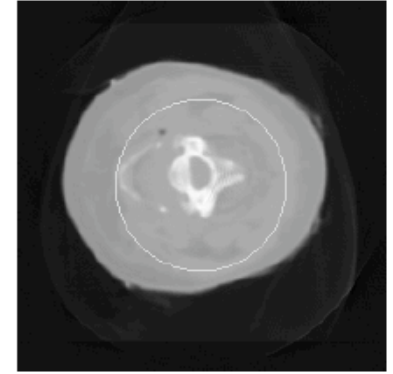

(c) $d=10$

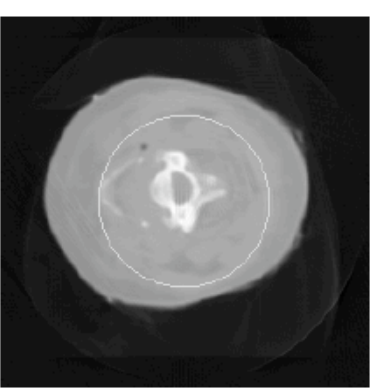

(d) $d=15$

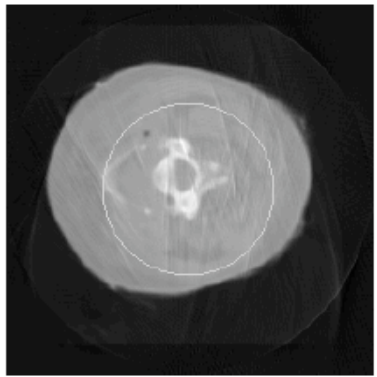

(e) $d=20$

Fig. (6). Irregular geometry results for the neck volume. (a) plots the NMSE for reconstructions of the data acquired from a deformed scanner over a range of deformation factors. The solid line depicts our reconstruction method that incorporates the irregular geometry, while the dotted line assumes a circular scanner geometry. Images (b)-(e) show the reconstructed slices using our method, along with the outline of the ROI used for computing the NMSE.

shows our scanner geometry for a variety of deformation factor values $(d=5,10,15,20)$. For each deformed scanner configuration, we reconstruct $\mathrm{CT}$ images and compare the results with the gold standard image shown in Fig. (4d).
Figs. (6 and 7) display the results of this experiment for both test volumes. The NMSE values for our reconstruction method are plotted as the solid line in (a). As a reference, (a) also plots the NMSE values that one would attain by 


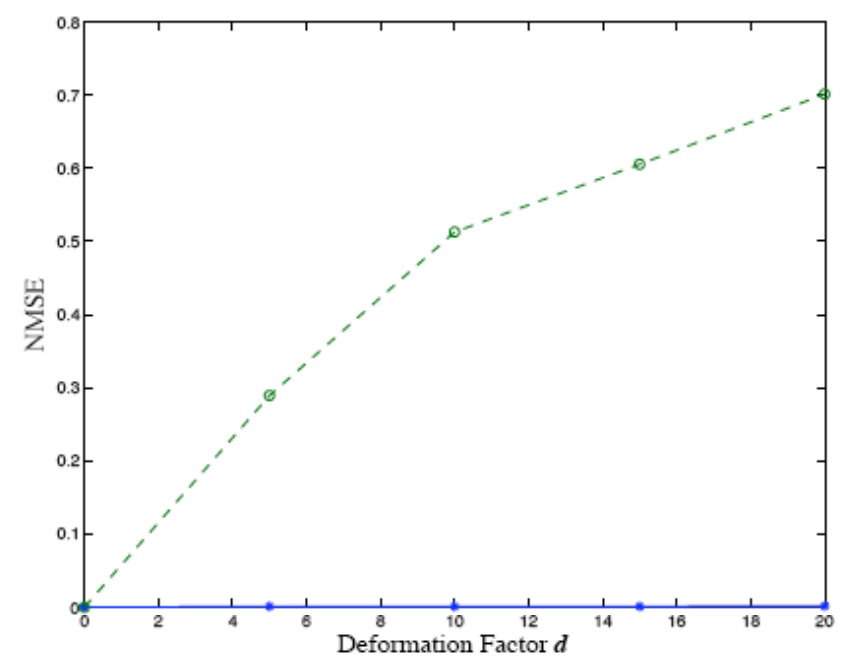

(a)

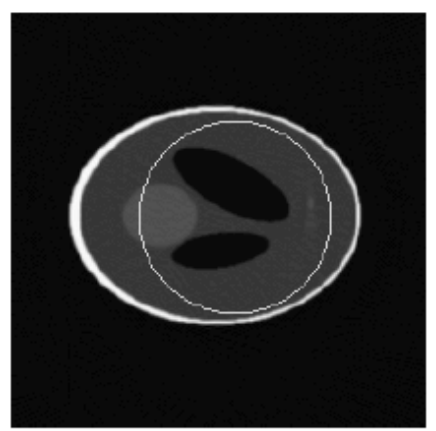

(b) $d=5$

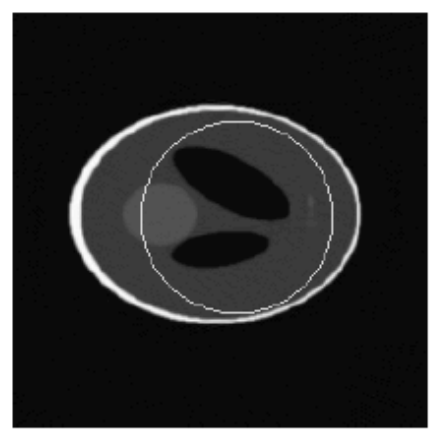

(c) $d=10$

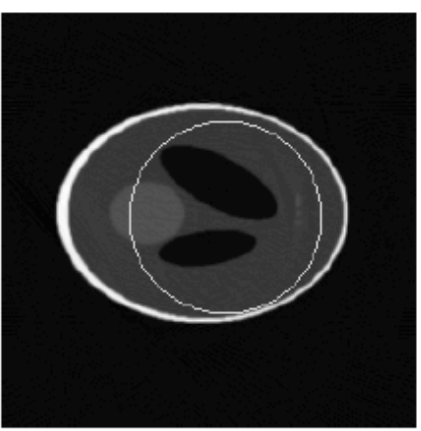

(d) $d=15$

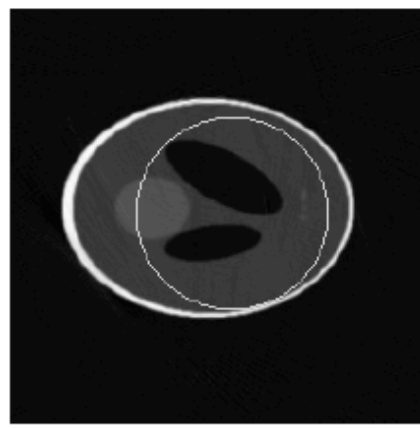

(e) $d=20$

Fig. (7). Irregular geometry results for phantom volume. (a) plots the NMSE for reconstructions of the data acquired from a deformed scanner over a range of deformation factors. The solid line (very close to the horizontal axis) depicts our reconstruction method that incorporates the irregular geometry, while the dotted line assumes a circular scanner geometry. Images (b)-(e) show the reconstructed slices using our method, along with the outline of the ROI used for computing the NMSE.

erroneously assuming the scanner was cylindrical (dotted line). Compared to the reference reconstructions, the NMSE values of our flexible reconstruction method are small and stable over the entire range of scanner deformations we simulated. Figs. (6b-e and 7b-e) show some corresponding reconstructed images.

The reference NMSE values for the phantom dataset are markedly larger than those for the neck dataset. After performing some experiments (not reported here), we believe the difference results from the image content; the phantom dataset contains many high-contrast edges, while the neck dataset tends to be more smooth. Using the wrong geometry to reconstruct the images causes geometrical distortion in the reconstructed images (see section 3.2). When the highcontrast edges of the phantom image are distorted, they produce large intensity disparities and increase the NMSE abruptly.

\subsection{Geometric Error Test}

The second experiment tests the sensitivity of the reconstruction method with respect to positional errors of the $\mathrm{CT}$ scanner. Unlike a traditional CT scanner, the flexible CT scanner is not fixed. Therefore, it is important to measure the correct positions of the emitters and detectors in the scanner.
Let us here distinguish between two geometrical configurations. One is the true acquisition geometry when the data is acquired. The other is the geometry that is used in the reconstruction process, denoted reconstruction geometry. Ideally, these two geometries should be the same, but in the real world, we expect that they will differ somewhat because of measurement error. Thus, a reconstruction method should be capable of producing stable outputs within some limit of measurement error. To simulate the geometric error, we use a different geometry for reconstruction than was used for acquisition. As a result, the reconstructed images contain geometrical artifacts.

The results for the geometric error test for the Visible Female volume are shown in Table 1 and Fig. (8). Table 1 lists the NMSE for combinations of varying degrees of acquisition and reconstruction deformation. The degree of deformation in the acquisition geometry, denoted $d_{a}$, is listed along the horizontal axis, and the degree of deforma-tion in the reconstruction geometry, denoted $d_{r}$, is listed along the vertical axis. The shape of the deformations is the same for both geometries, but the degree of the deformation changes as you move along an axis. The diagonal elements represent the cases when the acquisition and reconstruction geometry are the same (In fact, the diagonal holds the results from the first experiment). Recall that changing the deformation 
Table 1. NMSE Results for Geometric Error Experiment on the Visible Female Neck Volume

\begin{tabular}{|l|c|c|c|c|c|c|}
\hline & & \multicolumn{5}{|c|}{ Acquisition Geometry $\left(\boldsymbol{d}_{\boldsymbol{a}}\right)$} \\
\hline \multirow{3}{*}{ Reconstruction Geometry $\left(d_{r}\right)$} & & 0 & 5 & 10 & 15 & 20 \\
\hline & 0 & 0 & 0.0055 & 0.0144 & 0.0413 & 0.0711 \\
\cline { 2 - 9 } & 5 & 0.0050 & 0.0002 & 0.0047 & 0.0118 & 0.0354 \\
\cline { 2 - 9 } & 10 & 0.0125 & 0.0045 & 0.0003 & 0.0042 & 0.0106 \\
\cline { 2 - 8 } & 15 & 0.0206 & 0.0112 & 0.0042 & 0.0005 & 0.0041 \\
\cline { 2 - 8 } & 20 & 0.0306 & 0.0197 & 0.0112 & 0.0047 & 0.0015 \\
\hline
\end{tabular}

Table 2. NMSE Results for Geometric Error Experiment on the Phantom Volume

\begin{tabular}{|l|c|c|c|c|c|c|}
\hline & & \multicolumn{4}{|c|}{ Acquisition Geometry $\left(\boldsymbol{d}_{\boldsymbol{a}}\right)$} \\
\hline \multirow{3}{*}{ Reconstruction Geometry $\left(d_{r}\right)$} & & 0 & 5 & 10 & \multicolumn{2}{|c|}{15} \\
& 0 & 0 & 0.2892 & 0.5126 & 0.6054 & 0.7013 \\
\cline { 2 - 7 } & 5 & 0.1101 & 0.0006 & 0.3162 & 0.5207 & 0.6273 \\
\cline { 2 - 7 } & 10 & 0.2239 & 0.0877 & 0.0007 & 0.3336 & 0.5384 \\
\cline { 2 - 7 } & 15 & 0.2908 & 0.2012 & 0.0708 & 0.0008 & 0.3124 \\
\cline { 2 - 7 } & 20 & 0.3066 & 0.2770 & 0.1794 & 0.0602 & 0.0016 \\
\hline
\end{tabular}

factor by 5 moves the devices $9.3 \mathrm{~mm}$ (on average). Hence, the emitter and detector device positions are displaced from their true locations by an average of $1.86 \mathrm{~mm}$ for each unit change in deformation factor.
Fig. (8a) plots the data in Table $\mathbf{1}$ as a surface. The farther away from the diagonal, the larger the NMSE. To show more clearly the results of the test, Fig. (8b) plots the NMSE for a fixed acquisition geometry $\left(d_{a}=10\right.$, corresponding to

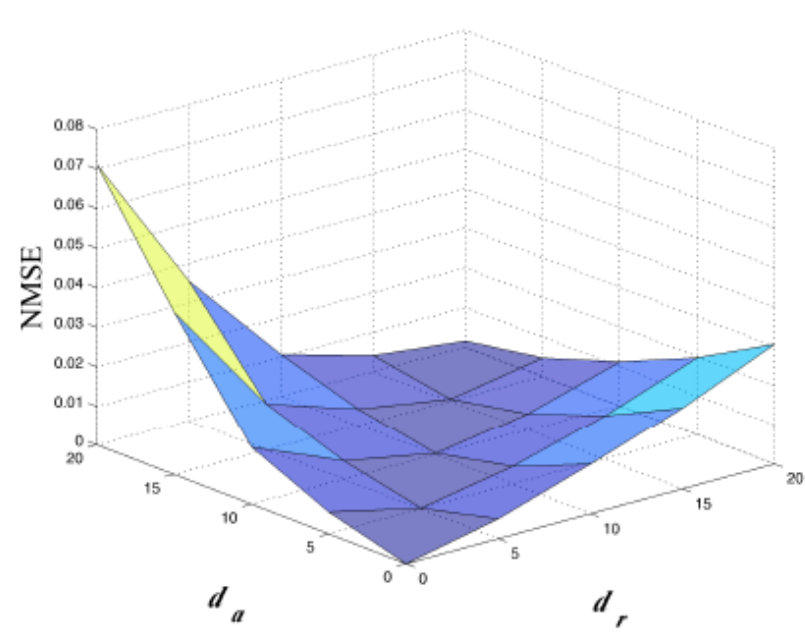

(a)

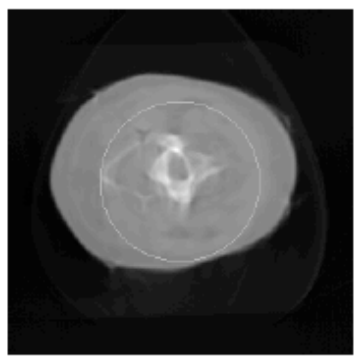

(c) $d_{r}=5$

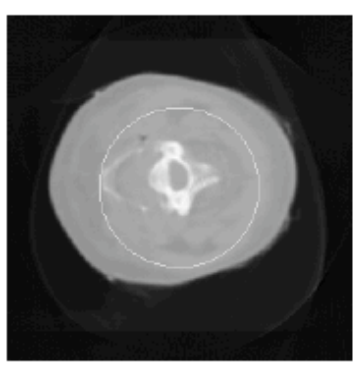

(d) $d_{r}=9$

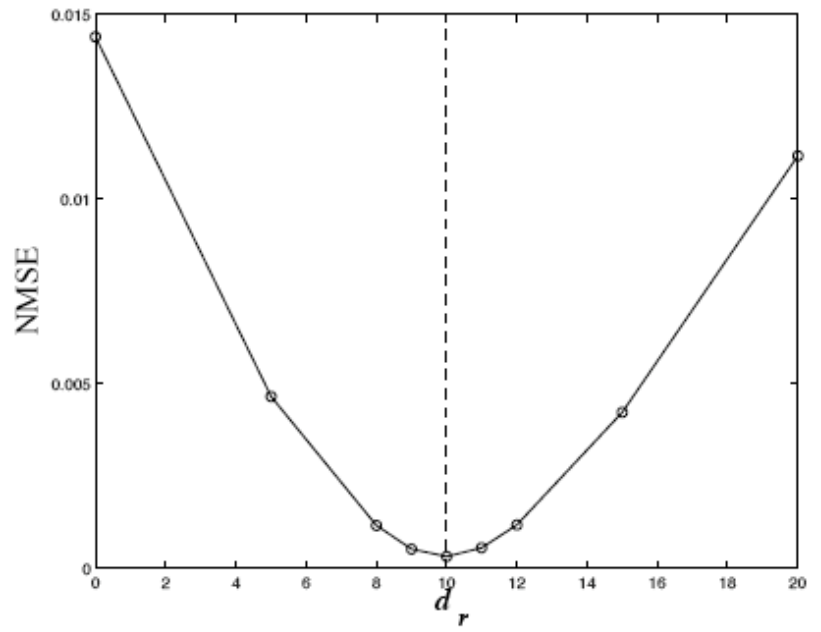

(b)

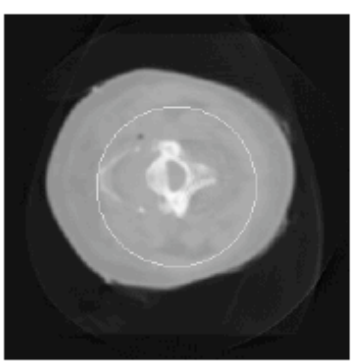

(e) $d_{r}=11$

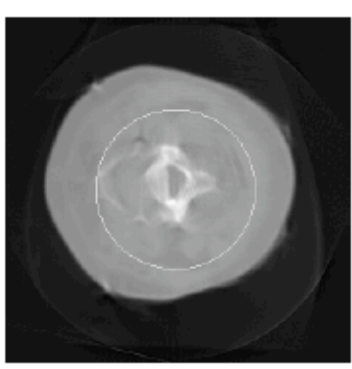

(f) $d_{r}=15$

Fig. (8). Geometric-error results for neck volume. (a) is a surface plot of the data in Table 1. (b) shows a cross-section of that surface (with additional data points) corresponding to an acquisition geometry deformation factor of $d_{a}=10$. The vertical dashed line shows where the reconstruction geometry matches the acquisition geometry. Images (c)-(f) show some of the reconstructed slices for $d_{a}=10$, along with the ROI used for computing the NMSE. 


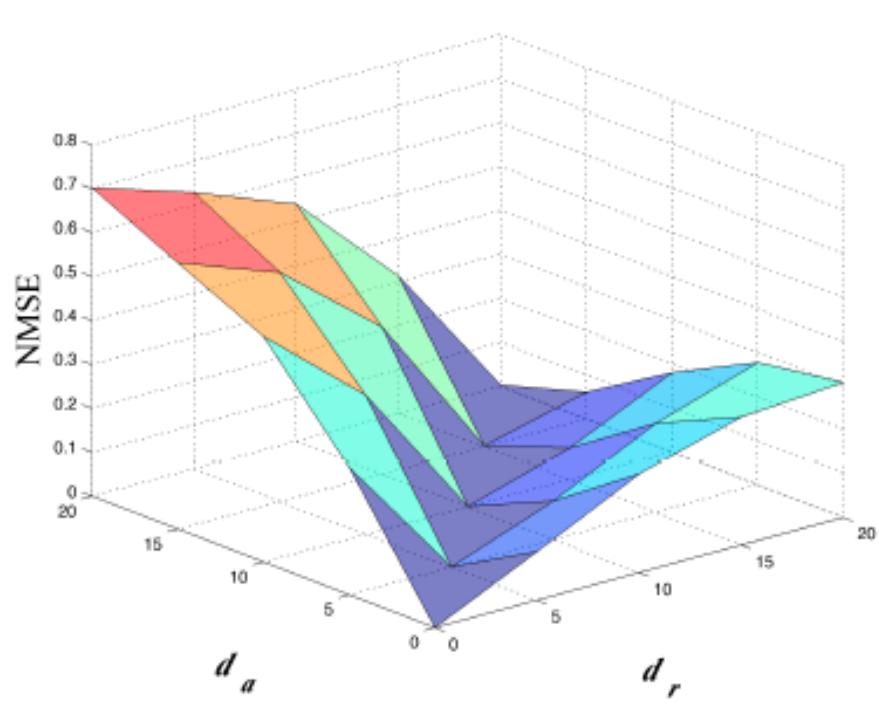

(a)

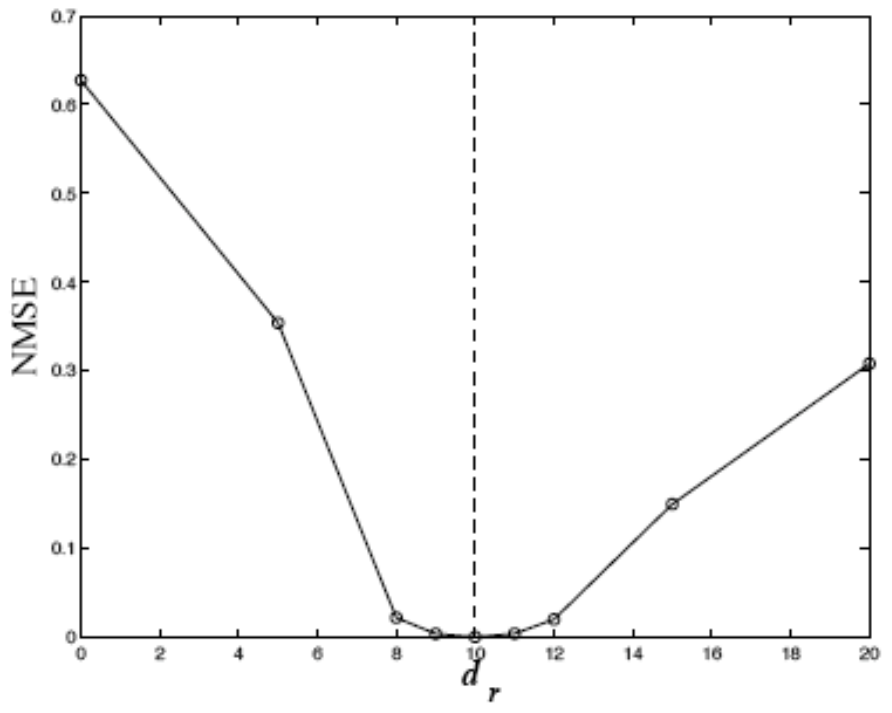

(b)

Fig. (9). Geometric-error results for phantom volume. (a) is a surface plot of the data in Table 2. (b) shows a cross-section of that surface (with additional data points) corresponding to an acquisition geometry deformation factor of $d_{a}=10$. The vertical dashed line shows where the reconstruction geometry matches the acquisition geometry.

the column with heading " $10 "$ in Table 1). The NMSE is more stable near $d_{r}=10$, but increases as the geometric error is increased. The corresponding reconstructed images are shown in Figs. (8c-f). In (c) and (f), we can see that the size and shape of the neck changes as the reconstruction geometry deviates significantly from the acquisition geometry.

Table 2 shows the same information as Table 1, but for the phantom test volume. Similarly, Fig. (9) plots the results of the geometric error test for the phantom volume, again as a surface and cross-section for $d_{a}=10$.

Figs. (10b-f) show some sample reconstructed images of the phantom volume. For comparison, Fig. (10a) shows a comparable fan-beam reconstruction with the same radius as our flexible scanner, $1^{\circ}$ detector spacing, and projections

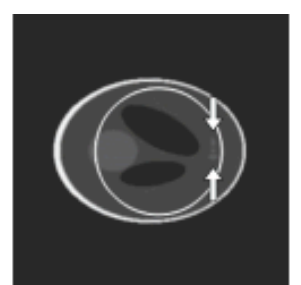

(a) Fan-beam

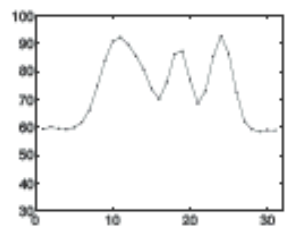

(g) Fan-beam

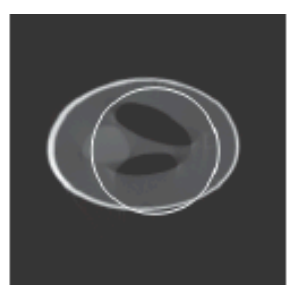

(b) $d_{r}=5$

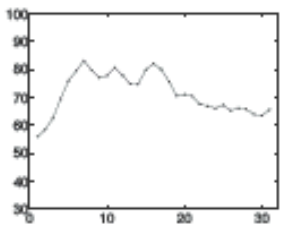

(h) $d_{r}=5$

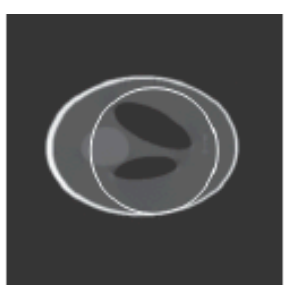

(c) $d_{r}=8$

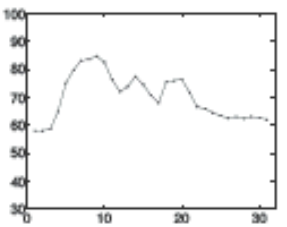

(i) $d_{r}=8$

taken at $1^{\circ}$ increments for a full $360^{\circ}$. Note that this is approximately four times the number of samples as our flexible scanner, since our emitter and detector spacing is $2^{\circ}$. We can get an impression of how spatial resolution degrades with geometrical error from the plots shown in Figs. (10g-l). Between the two arrows in Fig. (10a) are three small ellipses, high-resolution features of the phantom. Each plot in (g)-(1) graphs the cross-section of intensity values through those ellipses. The fidelity of the three peaks becomes compromised for large geometrical error $\left(d_{r}=5\right.$ or 15$)$, but is stable for smaller distortions ( $d_{r}=8$ and 12$)$.

\subsection{X-Ray Cone Beam Coverage}

In our final experiment, we test how sensitive our reconstruction method is to issues regarding $\mathrm{x}$-ray cone beam

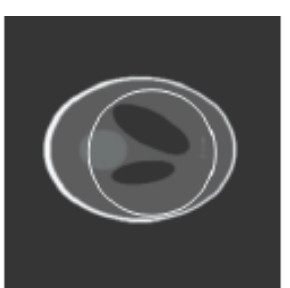

(d) $d_{r}=10$

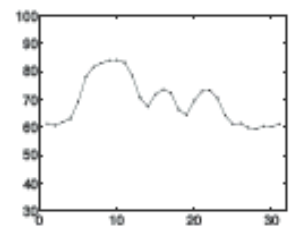

(j) $d_{r}=10$

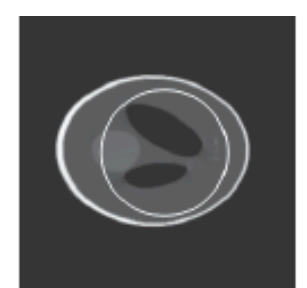

(e) $d_{r}=12$

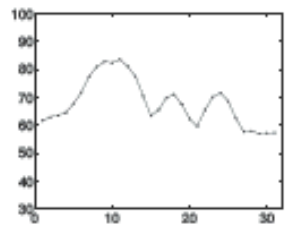

(k) $d_{r}=12$

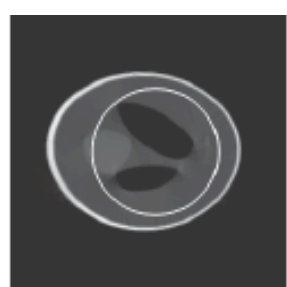

(f) $d_{r}=15$

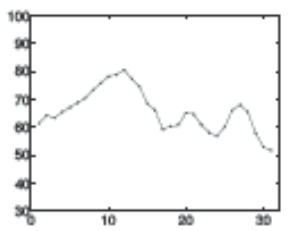

(1) $d_{r}=15$

Fig. (10). Geometric-error reconstructions for phantom volume. (a) shows a reconstruction of the phantom from a fan-beam CT scanner with detectors spaced $1^{\circ}$ apart and projections taken at $1^{\circ}$ increments. Images (b)-(f) show reconstructions using a range of reconstruction geometries $\left(d_{r}\right)$ on data acquired with $d_{a}=10$. The ROI used for computing the NMSE is outlined in (a)-(f). The arrows in (a) point to three high-resolution features in the phantom. The graphs in (g)-(I) plot the intensity profile of each reconstruction through the high-resolution features. 


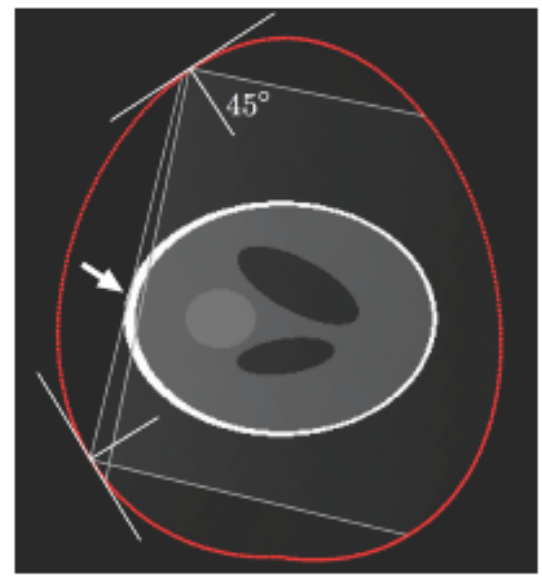

(a) Scanner

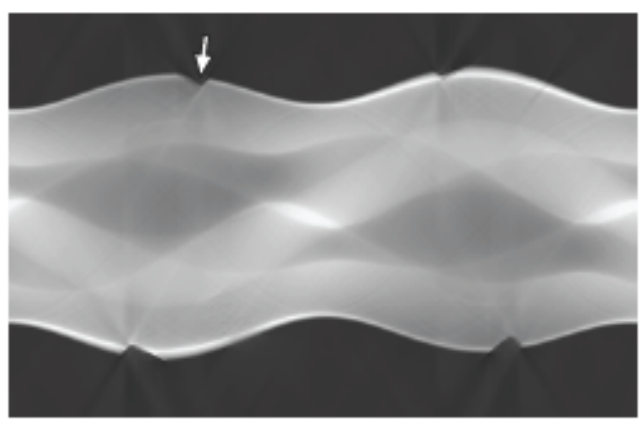

(b) Sinogram

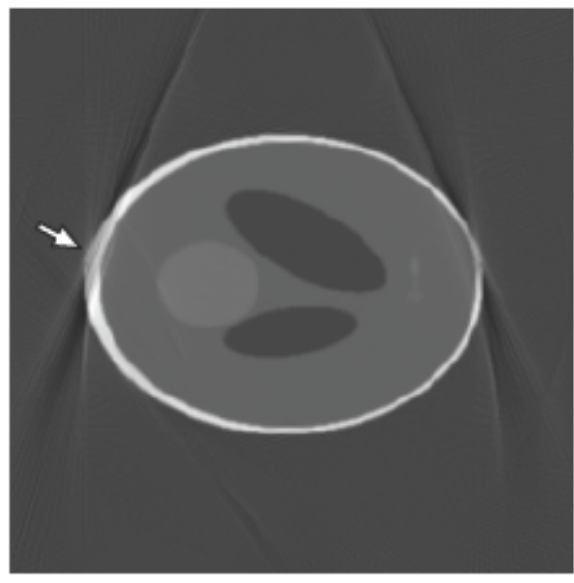

(c) Reconstruction

Fig. (11). Effect of a $90^{\circ}$ cone angle. The arrow in (a) shows one edge that is not captured by any tangential ray sums. The two x-ray cones that come closest to capturing the edge are also shown in (a). As a result, data is missing from the x-ray transform, as indicated by the arrow in (b). The arrow in (c) points to where the corresponding reconstructed edges are blurred.

coverage. If the scanner is too close to the subject, critical regions of the x-ray transform space can be under-sampled, or not sampled at all. Here we show two cases where reconstruction artifacts result from: (a) a reduced cone angle, and (b) the scanner being too close to the subject.

Fig. (11) shows a scenario where the cone angle is reduced so that the full apex angle is $90^{\circ}$ (instead of $120^{\circ}$, as in the previous experiments). The reduced field of view causes part of the x-ray transform space to be undersampled (or not sampled at all), as shown in Fig. (11b). The resulting reconstruction artifact is shown in Fig. (11c), where some edges are blurred, with streaks appearing tangent to the highcontrast edges.

The effect of the scanner being too close to the patient is similar to the cone-angle effect above. Fig. (12) shows a case in which the scanner passes very near the anatomy. Despite the fact that the cone angle is $120^{\circ}$, data appears to be missing from the $\mathrm{x}$-ray transform, as shown in Fig. (12b). Again, the reconstructed image in Fig. (12c) shows blurred edges in the regions where the scanner was closest. Other streak artifacts are also apparent.
These test cases point to the rule of thumb that ray sums tangent to image edges are needed in order to reconstruct the edge in a stable and accurate manner [20].

\section{DISCUSSION}

There are many challenges that must be overcome before a flexible and portable CT scanner can be built. In what follows, we outline the most obvious issues and suggest some possible solutions.

The resolution of the reconstructed images depends on the number of emitters and detectors, as well as their position in 3-space. The spacing and arrangement of these devices needs to be considered when designing a scanner. The required resolution will dictate the emitter and detector distributions. Moreover, our simulations suggest that scannersubject spacing also affects image resolution and clarity. If possible, the scanner design should incorporate a guide for setting the appropriate scanner-patient spacing. For example, a cushion sleeve could help when deploying the scanner around a patient.

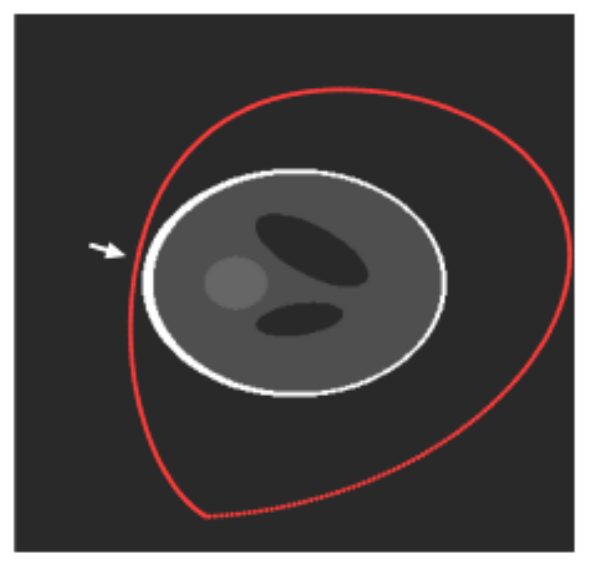

(a) Scanner

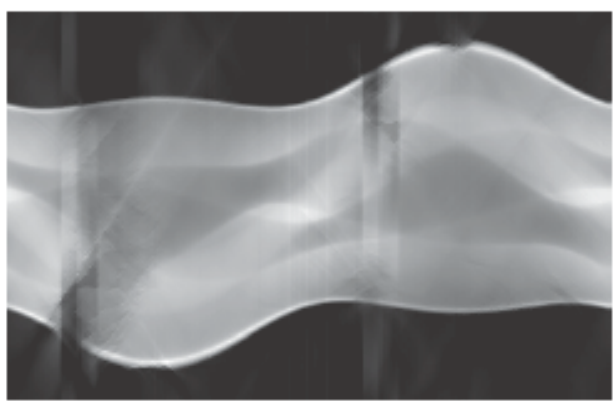

(b) Sinogram

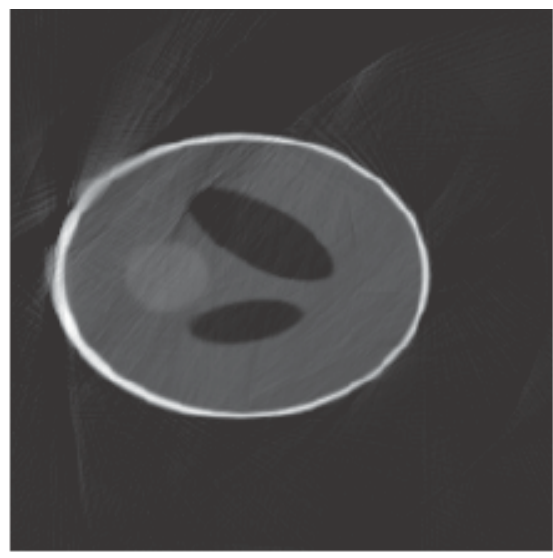

(c) Reconstruction

Fig. (12). Effect of scanner being too close to the subject. The arrow in (a) shows where the scanner passes close to the phantom. As a result, data is missing from the $\mathrm{x}$-ray transform, as shown in (b). Artifacts are visible in (c). 
The beam coverage simulations illustrate that the scanner should try to detect geometries that cause extreme undersampling before an x-ray dose is delivered. The scanner's geometrical conformation must be recorded at acquisition time, so can be used to detect unfavourable geometry at that time.

How the geometry of the scanner impacts the quality of the images is a complex topic. There are myriad image metrics, as well as many ways to deform the scanner. What particular artifacts are tolerable -- and what image deficiencies render the image useless -- will depend on the application. A fuller understanding of image sensitivity to geometrical variations needs to be undertaken. For example, a sensitivity analysis on the discernibility of a small image feature (like the tiny ellipses in Fig. 10) might help establish guidelines for what geometries are tolerable when detecting vertebral fractures.

Calibration of the detectors can be done with an "air scan" once a day before dispatching the unit for use. The flexible scanner can be placed into a shielded cylindrical frame with a well-characterized x-ray source that moves. As the source moves, the detectors collect flux data that can be used to calibrate their sensitivities, including directional sensitivity. Once the detectors are calibrated, they can be used to characterize the x-ray emitters. Each emitter is turned on and its $\mathrm{x}$-ray flux is measured by the detectors.

Each CNT has to be in a vacuum for it to emit x-rays. The prospect of encasing each emitter in its own vacuum container poses a forbidding technical challenge. However, the final design will certainly be a compromise between performance and cost. A fully flexible blanket that is capable of flexing in multiple degrees-of-freedom requires each source to be vacuum packaged individually and connected through hinges. However, we expect that most of the functionality of such a scanner could be realized in a scanner that is more constrained. For example, it is envisioned that a 1-D array of emission sources could be vacuum packaged together as one unit, and multiple units connected by hinges to provide flexibility in 1-degree-of-freedom. This imaging system is more economical and presents less of a technological challenge, while providing sufficient capability to conform to large critical body structures such as head, neck, and chest.

The structural integrity of carbon nanotubes degrades over time. This degradation results in reduced x-ray flux, and hence factors prominently in the use of CNTs for our application. Moreover, the nature of the degradation processes are not well understood. More research is needed to address this issue.

It is not clear how much $\mathrm{x}$-ray photon flux we can expect from CNTs. Physical experiments have produced some compelling results, with exposure times on the order of 10 seconds $[1,11]$. If the flux is too low, then the image quality will suffer with a low SNR. This low flux issue can be mitigated by longer scan times, but then patient motion becomes more of a problem. Alternatively, a stronger electric field can be used to increase flux, but might cause interference with nearby emitters and detectors. Investigations into this effect are ongoing [21].
Work is currently underway to build $\mathrm{x}$-ray detectors that operate in two modes: integration mode, and photoncounting mode [22]. All clinical CT scanners use integrating detectors, appropriate for high photon flux applications. Photon-counting detectors are far more sensitive when the photon flux is low, but become saturated when the flux increases. Dual-mode detectors are capable of both integration mode and photon-counting mode. These dual-mode detectors might solve the low-flux issues potentially facing CNT x-ray emitters.

The geometrical conformation of the scanner sheet (the positions of the emitters and detectors) is an essential input to the reconstruction software outlined in this paper. ShapeTape, a motion-tracking device designed to track the curves traced by a thin strip in 3-D, seems like an obvious choice for this application. While ShapeTape is designed to report shape in a high-dimensional space, its ability to be used as an absolute positioning system is problematic [23]. However, one can imagine that a second motion-tracking system could be used to augment the shortcomings of the ShapeTape. For example, a grid of motion-tracking markers on the scanner sheet could give a good absolute position, while the ShapeTape acts like an interpolator, giving information about how the sheet bends between the markers. The absolute motion-tracking system probably cannot be line-of-sight based (like an optical motion-tracking system), so is more likely to be an electromagnetic tracking system. However, these tracking systems can experience interference in the presence of metal objects, which might pose a problem for many scenarios involving traffic accidents. Another option to measure scanner geometry is to use radio-frequency (RF) ranging. This technology is similar to the global positioning system (GPS), except that our system would have to use higher frequencies, and our synchronization would be easier because the sensors could be connected by wires.

A study based on the simulation of a flexible single-slice (2-D) scanner demonstrated that geometrical errors could be corrected by fine-tuning the scanner geometry to minimize the image entropy [24]. A 3-D implementation of the idea, while workable in principle, would involve a more challenging and computationally expensive optimization problem.

If the x-ray cone apex angles are too small, or if the scanner is too close to the patient, the resulting $\mathrm{x}$-ray transform will be missing data. However, this issue might be addressed by a reprojection method. In our reconstruction method, the density of samples in the x-ray transform space is computed as part of the process. Hence, we have knowledge of where data is missing. A reprojection method [25] could be used to estimate the missing data and generate better images. Further work is needed in this arena.

Finally, there will certainly be safety issues with an x-ray device that can be deployed outside of a controlled environment. Obviously, measures need to be taken to prevent x-ray exposure to those who deploy and operate the scanners.

\section{CONCLUSION}

The geometrically adaptive reconstruction method outlined in this paper effectively incorporates the irregular 
shape of the flexible CT scanner. Despite rather large deformations, the reconstructed images remain relatively accurate. Robustness tests with respect to measurement errors in scanner geometry show that emitter and detector displacements of up to $4 \mathrm{~mm}$ have little impact on the quality of the reconstructed images.

Before a flexible and portable CT scanner can be built, however, there are many technical challenges -- both known and unforeseen -- that must be overcome. It is our view that many of these issues are engineering problems, and can be solved. Materialization of a flexible and portable scanner will most likely evolve considerably during development.

\section{CONFLICT OF INTEREST}

None declared.

\section{ACKNOWLEDGEMENT}

This work was supported by funding from the Natural Sciences and Engineering Research Council of Canada (NSERC), the Canada Foundation for Innovation, and the Ontario Innovation Trust.

\section{REFERENCES}

[1] Zhang J, Yang G, Cheng Y, et al. Stationary scanning X-ray source based on carbon nanotube field emitters. Appl Phys Lett 2005; 86: 184104.

[2] Sinha N, Yeow JTW. Carbon nanotubes for biomedical applications. IEEE Trans Nanobiosci 2005; 4(3): 180-95.

[3] Iijima S. Helical microtubules of graphitic carbon. Nature 1991; 354: 56-8.

[4] Rinzler AG, Hafner JH, Nikolaev P. Unraveling nanotubes: Field emission from an atomic wire. Science 1995; 269(5230): 1550-3.

[5] de Heer WA, Châtelain A, Ugarte D. A carbon nanotube fieldemission electron source. Science 1995; 270(5239): 1179-80.

[6] Chernozatonskii LA, Gulyaev YV, Kosakovskaja ZJ, et al. Electron field emission from nanofilament carbon films. Chem Phys Lett 1995; 233: 66-8.

[7] Bonard JM, Salvetat JP, Stöckli T, Forró L, Châtelain A. Field emission from carbon nanotubes: perspectives for applications and clues to the emission mechanism. Appl Phys A 1999; 69(3): 24554

[8] Sugie H, Tanemura M, Filip V, Iwata K, Takahashi K, Okuyama F. Carbon nanotubes as electron source in an X-ray tube. Appl Phys Lett $2001 ; 78(17): 2578-80$.
[9] Fowler RH, Nordheim L. Field emission from metallic surfaces. Proc R Soc London A 1928; 119: 173.

[10] Sun J, Zhang Z, Hou S, et al. Work function of single-walled carbon nanotubes determined by field emission microscopy. Appl Phys A 2002; 75(4): 479-83.

[11] Zhang J, Yang G, Lee YZ, Chang S, Lu JP, Zhou O. Multiplexing radiography using a carbon nanotube based x-ray source. Appl Phys Lett 2006; 89: 064106.

[12] Quan E, Lalush DS. Evaluation of hexgonal and square geometries for motion-free arrayed-source x-ray micro-CT. In: Proc. of the IEEE International Symposium of Biomedical Imaging (ISBI'07), 2007; pp. 221-4.

[13] Orchard J, Yeow JTW. Toward a flexible and portable CT scanner. In: Metaxas D, Axel L, Fichtinger G, Székely G, Eds. Proceedings of Medical Image Computing and Computer Assisted Intervention (MICCAI), vol. LNCS 5242. Springer, September 2008; pp. 18895.

[14] Ramotar A, Orchard J. General geometry CT reconstruction. In: Arabnia HR, Ed. Proceedings of the International Conference on Image Processing and Computer Vision (IPCV'06). vol. 1, June 2006; pp. 95-9.

[15] Defrise M, Kinahan PE, Townsend DW. Exact and approximate rebinning algorithms for 3-D PET data. IEEE Trans Med Imaging 1997; 16(2): 145-58.

[16] Parzen E. On estimation of a probability density function and mode. Annal Math Stat 1962; 33(3): 1065-76.

[17] Defrise M, Liu X. A fast rebinning algorithm for 3D positron emission tomography using John's equation. Inverse Probl 1999; 15(4): 1047-65.

[18] Mazin SR, Pelc NJ. A fast 3D reconstruction algorithm for inversegeometry CT based on an exact PET rebinning algorithm, In: Hsieh J, Flynn MJ, Eds. Proceedings of SPIE Med Imaging. San Diego 2007.

[19] Abramoff MD, Viergever MA. Computation and visualization of three dimensional motion in the orbit. IEEE Trans Med Imaging 2002; 21(4): 296-304.

[20] Natterer F, Wübbeling F. Mathematical methods in image reconstruction. SIAM. UK: Cambridge University Press 2001.

[21] Sinha N, Yeow JTW, Jaffray DA. Experimental investigation of the crosstalk phenomenon and current stability in a carbon nanotube array. In: Proceedings of 7th IEEE International Conference on Nanotechnology, Hong Kong, August 2007.

[22] Goldan AH, Hadji B, Karim KS, et al. A counting and integrating pixel readout chip for amorphous selenium direct radiation detectors for medical imaging applications, In: SPIE International Medical Imaging Symposium 2009; vol. 7258.

[23] Baillot Y, Eliason JJ, Schmidt GS, et al. Evaluation of the Shape Tape tracker for wearable, mobile interaction. In: Proceedings of the IEEE Virtual Reality 2003.

[24] Orchard J, Ramotar A. Autocorrecting reconstruction for flexible CT scanners. In: Proceedings of the IEEE International Symposium of Biomedical Imaging (ISBI'07), April 2007; pp. 804-807.

[25] Kinahan PE, Rogers JG. Analytic 3D image reconstruction using all detected events. IEEE Trans Nuclear Sci 1989; 36(1) 964-8. 